\title{
Satellite Images Recovery Based on Wiener filter
}

\author{
Xiao Jingxin ${ }^{1, a}$, Xiong Zhuang ${ }^{1, a}$, Zhang Zhichun ${ }^{1,}$, Zhang feng $^{1, \text { a }}$, Xu Wen $^{1 \text {, a }}$ \\ ${ }^{1}$ Air Force Aviation University, ChangChun 130022, China \\ aemail: xjx7810@163.com
}

Keywords: Motion Blur; Image De-noising; Image restoration; Wiener filter

\begin{abstract}
As for the problem that ground scenery image interfered causes its quality decreased, and in order to get high-quality data ground scenery, in this paper, firstly analyze the main factors of image degradation and then we adopt wiener filtering to recover image which is interfered by movement blurred and noise. The results show the effectiveness of method.
\end{abstract}

\section{Introduction}

We will use satellites to get geographic information data in the production scene database, But this image always exist the problem of quality decreased. In order to improve image quality and the focus landmark sharpness, in this paper, we adopt the image restoration algorithm to image restoration.

Motion blurring and image noise are important factor affecting the image quality. Due to the relative motion between the camera and the object, it often can cause image blur and the noise is unavoidable. For aeronautical satellite image, these factors will bring serious consequences.

In recent years, the image restoration technology has got great development, HuntB.R.[1] adopts Phihps's method, getting the constrained least square method. Rudin vosher and Fatemi first put forward the total variation model[2,3]. This model de-noises effectively and protects the image edge at the same time, so it may be widely admired both at home and abroad. The total variation model and other methods combination used in image processing has become a hot topic in academic research in recent years. Such as it combined with neural network are used in image restoration[4,5].

\section{The main factor image quality decline}

Blur: The main of image blurred is the imaging system focusing improperly or the relative movement of between camera and target. These belong imaging system defects and the atmospheric turbulence at long exposure. Image displacement is an important factor of image blurring. In the camera exposure time the relative movement between target image and imaging media called image displacement. Image displacement cause the degradation of image quality and it will make image produce two kinds of distortion which are shape and size of the image distortion and image brightness distortion. The distortion of the image will lead to image resolution decreased. When the image displacement is large, the clarity of images will reduce and image will be blurred. As shown in Figure 1.

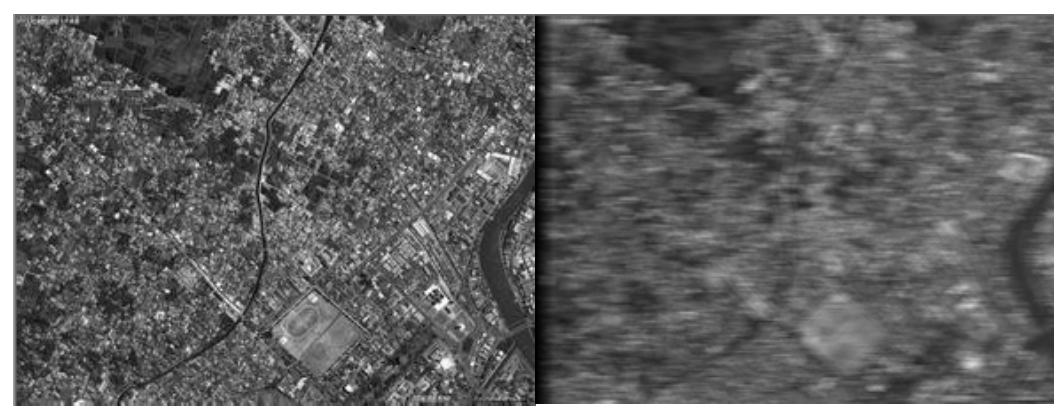

Fig.1. the motion blur image

Noise: The aviation image will generate a large amount of noise in the collection, quantization 
and transmission process. The image acquisition equipment is affected by many factors in the image collection process, such as environmental temperature etc. For example, when the CCD camera sensor temperature is too high, it may produce a large of noise. In addition, the uncertain factors in the process of image transmission always lead to of noise. For example, when the wireless network transmission image, lightning, atmospheric disturbances and other uncertain factors often cause image degradation. Because of noise and other uncertain factors, it brings certain difficulties to the recognition of image information. So it is very important to study noise model. Image noise divided into many types which classified by the relations of noise and signal has additive noise and multiplicative noise and by the probability density function has Gaussian noise and impulse noise. Because of the Gaussian white noise is widely present in degraded image, in this paper we mainly research the image which is polluted by Gaussian white noise. As shown in Figure 2.

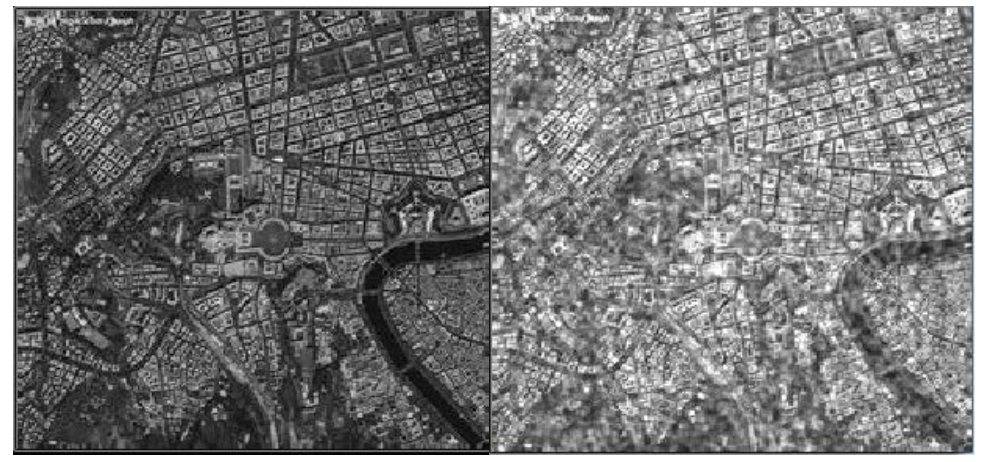

Fig.2. the noise image

\section{Image evaluation standard}

Image quality refers to the people evaluation of image visual perception. With the rapid development of image restoration technology, whether checking the quality of restored image is objective has become the focus of attention.

Image quality assessment is divided into subjective and objective evaluation. Image subjective evaluation is to determine the image quality by people. Image Objective evaluation is to determine the image quality by some statistical characteristics and physical parameters. These are some common objective evaluation standards such as minimum mean square error and peak signal noise ratio which are defined as follows.

$$
\begin{aligned}
& M S E=\frac{1}{M \times N} \sum_{x=0}^{M-1} \sum_{y=0}^{N-1}[u(x, y)-\bar{u}(x, y)]^{2} \\
& P S N R=10 \log _{10}^{\frac{255^{2}}{M S E}}
\end{aligned}
$$

In Formula $1, M \times N$ is the size of image, $u(x, y)$ is the original image and $\bar{u}(x, y)$ is the evaluation image. By Formula 3 we can know that the MMSE is smaller and the peak signal noise ratio is larger, the evaluation image closer to the original image. In this paper the minimum mean square error and peak signal noise ratio are used for image quality evaluation standard.

\section{Image restoration algorithm}

Wiener filter is a filter put forward by Wiener in the 1940s. It is an adaptive MMSE (Minimum Mean Square Error) filter. The method of wiener filter is a statistical method. The Optimal criteria it using is based on the correlation matrix of image and noise. It will adjust the output of filter by local variance of image and the bigger local variance, the stronger smoothing effect of the filter.

Wiener filter is an image recovery method with restraint. It integrates the characteristics both degraded image and image noise. Suppose the original image is $f(x, y)$ and De-noised image is $\hat{f}(x, y)$. Wiener filtering will make the least of mean square error between original image and 
de-noised image. As shown in Formula 2.

$$
E\left\{[h(x, y)-\bar{h}(x, y)]^{2}\right\}=\min
$$

In Equation 1, $E$ is mathematical expectation, so wiener filter is also known as the minimum mean Variance filter. Wiener filtering is based on minimizing the statistical criterion, and the results obtained from wiener filtering are optimal average sense. We can use Fourier transform to deduce the principles of wiener filtering.

$$
F(u, v)=H_{w}(u, v) G(u, v)=\frac{1}{H(u, v)} \bullet \frac{|H(u, v)|^{2}}{|H(u, v)|^{2}+\gamma \frac{p_{n}(u, v)}{p_{f}(u, v)}} \bullet G(u, v)
$$

Equation 2 restoration generic constraint expressions recovery, its transfer function is:

$$
H_{w}(u, v)=\frac{1}{H(u, v)} \cdot \frac{|H(u, v)|^{2}}{|H(u, v)|^{2}+\gamma \frac{p_{n}(u, v)}{p_{f}(u, v)}}
$$

\section{Test results}

The experiments divided into two part, these are we use wiener filtering algorithm to recover the images with motion blur and noise.

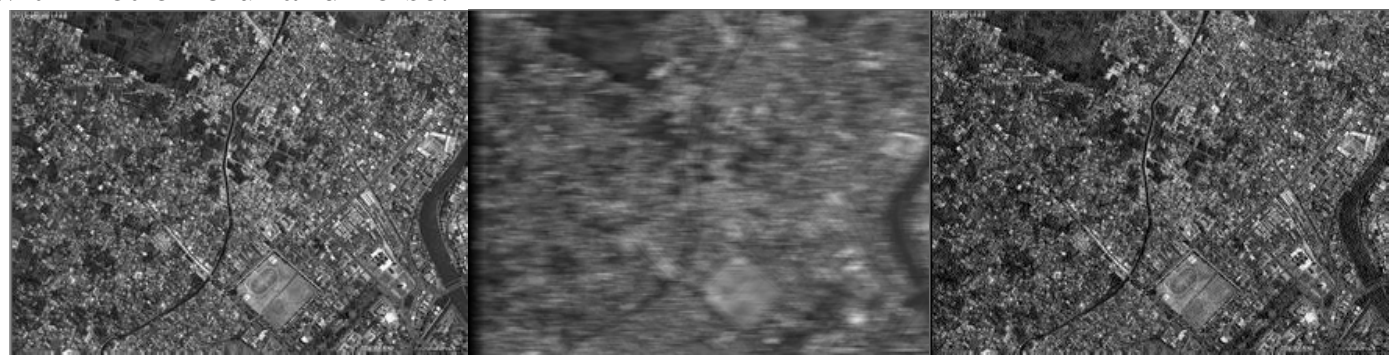

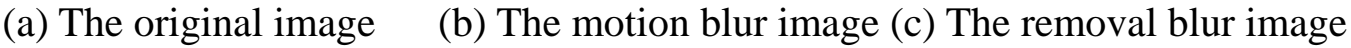

Fig.3. image remove blur experimental results

For movement blurred image in Fig.3, the value of MSE and PSNR are 1098.97 and 17.72 before recovery and after that the value of MSE and PSNR change to 248.67 and 24.17.

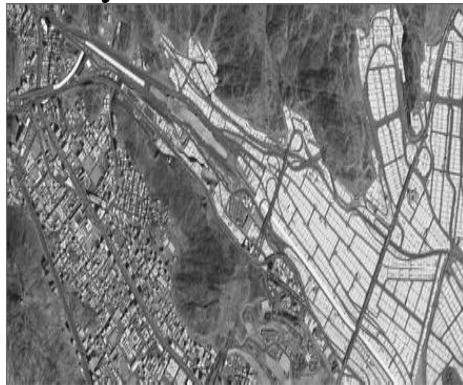

(a) The original image

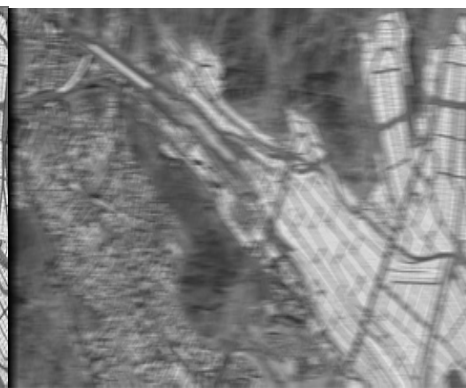

(b) The motion blur image (c) The removal blur image

Fig.4. image remove blur experimental results

For another image with higher brightness in Fig.4, the value of MSE and PSNR are 2459.12 and 14.22 before recovery and after that the value of MSE and PSNR change to 1374.24 and 20.75. From the results in Fig. 3 and Fig.4, the wiener filtering can effectively remove motion blur in the image. 


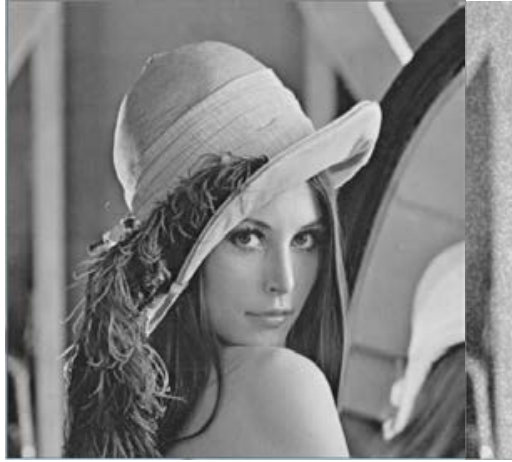

(a) The original image

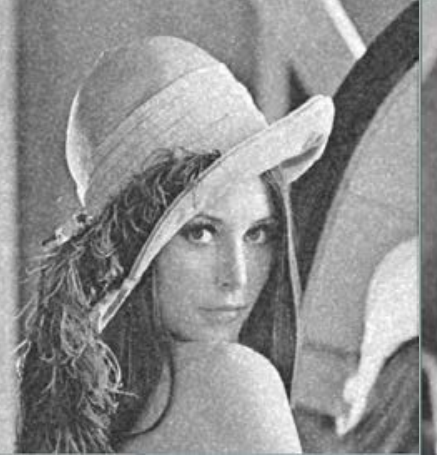

(b) The noise image

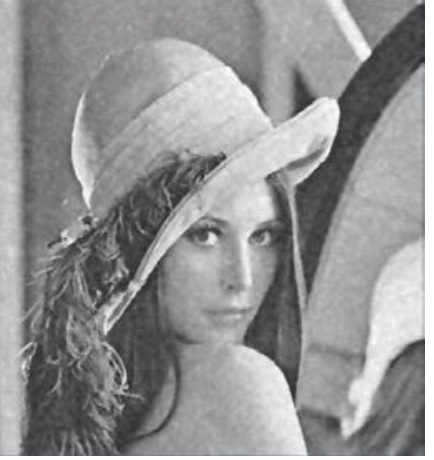

(c) The de-noise image

Fig.5. image de-noising experimental results

For the noise image, the value of MSE and PSNR are 476.09 and 17.63 before de-noising and after de-noising the value of MSE and PSNR change to 416.18 and 24.35. From the results, the wiener filtering can effectively de-noise in the image.

\section{Conclusion}

Motion blurring and image noise are important factors affecting image quality and unavoidable factors. The landscape images always with more texture information, In order to improve the clarity of the target, we must have the image post-processing. In this paper we put forward a method of image restoration algorithm based on Wiener filtering. This algorithm can eliminate motion blur and image noise effectively, and the experimental results prove the effectiveness of the algorithm.

\section{References}

[1] HuntB.R. The application of constrained least squares estimation to image restoration by digital computer[J].IEEETrans.ComPuters,1973,22(9):805-81.

[2] L.Rudin, S. Osher, E.Fatemi. Nonlinear total variation based noise removal algorithm[J]. Phys. D, 1992,60:259-26.

[3] C.W.Helstrom. Image Restoration by the Method of least Squares[J]. OPt. Soe. Amer. Mar, 1967,57(3):297-303.

[4] T.F.Chan, P. Mulet, On the convergence of the lagged diffusivity fixed point method in total variation image restoration[J]. SIAM J Numerieal Analysis,1999,36:354-36.

[5]Wu Chuan-yu, He Lei-ying, Design and Realization of Instructional RPPR-Robot[J], Research and Exploration in Laboratory.2007, 26(10)

[6] Nishiwaki K,Kagami S. High Frequency Walking Pattern Generation based on Preview Control of ZMP[C], IEEE International Conference on Robotics and Automation. 2006.

[7] Chen Nan and so on. For the field of industrial and highly interconnected, TI launched a new Sitara ARM9 microprocessor[J]. Global Electronics, 2010 (5) 86-87.

[8] Vision Heading Navigation Based on Navigation Curve [A]. Proceedings 2010 International Conference on Intelligent Computing and Integrated Systems[C]. 2010.

[9] Chen Nan and so on. For the field of industrial and highly interconnected, TI launched a new Sitara ARM9 microprocessor[J]. Global Electronics, 2010 (5) 86-87. 